\title{
ON HILL'S EQUATION WITH A DISCONTINUOUS COEFFICIENT
}

\author{
ILKAY YASLAN KARACA
}

Received 25 February 2002

\begin{abstract}
We research the asymptotic formula for the lengths of the instability intervals of the Hill's equation with coefficients $q(x)$ and $r(x)$, where $q(x)$ is piecewise continuous and $r(x)$ has a piecewise continuous second derivative in open intervals $(0, b)$ and $(b, a)(0<b<a)$.
\end{abstract}

2000 Mathematics Subject Classification: 34B30, 34L20, 47E05.

1. Introduction. We consider the second-order equation

$$
y^{\prime \prime}(x)+\{\lambda r(x)-q(x)\} y(x)=0, \quad-\infty<x<\infty,
$$

where $q(x)$ and $r(x)$ are real valued and all have period $a$. Also $q(x)$ is piecewise continuous, $r^{\prime \prime}(x)$ is piecewise continuous in $(0, b)$ and $(b, a)$ where $0<b<a$ and $r(x) \geq r_{0}(>0)$.

Those values of complex parameter $\lambda$, for which periodic or antiperiodic problem associated with (1.1) and the $x$-interval $(0, a)$ has a nontrivial solution $y(x, \lambda)$, are called eigenvalues. An equation giving the eigenvalues of the periodic or the antiperiodic problem can be found. For the purpose, after we use the Liouville transformation

$$
t=\int_{0}^{x} r^{1 / 2}(u) d u, \quad z(t)=r^{1 / 4}(x) y(x),
$$

the periodic boundary value problem becomes the boundary value problem

$$
\begin{gathered}
\ddot{z}+\{\lambda-Q(t)\} z(t)=0, \quad 0 \leq t \leq A, \\
z(A)=\sigma z(0), \quad \sigma \dot{z}(A)+\rho z(A)=\dot{z}(0)+\tau z(0)
\end{gathered}
$$

and the antiperiodic boundary value problem becomes the boundary value problem

$$
\begin{gathered}
\ddot{z}+\{\lambda-Q(t)\} z(t)=0, \quad 0 \leq t \leq A, \\
z(A)=-\sigma z(0), \quad \sigma \dot{z}(A)+\rho z(A)=-\dot{z}(0)-\tau z(0),
\end{gathered}
$$


where

$$
\begin{aligned}
r(x) & =\left\{\begin{array}{l}
r_{1}(x), \quad \text { if } 0 \leq x<b, \\
r_{2}(x), \quad \text { if } b<x \leq a,
\end{array}\right. \\
Q(t) & =\left\{\begin{array}{l}
\frac{q(x)}{r_{1}(x)}-r_{1}^{-3 / 4}(x)\left\{r_{1}^{-1 / 4}(x)\right\}^{\prime \prime}, \quad \text { if } 0 \leq t<B, \\
\frac{q(x)}{r_{2}(x)}-r_{2}^{-3 / 4}(x)\left\{r_{2}^{-1 / 4}(x)\right\}^{\prime \prime}, \quad \text { if } B<t \leq A,
\end{array}\right. \\
A & =\int_{0}^{a} r^{1 / 2}(u) d u, \quad B=\int_{0}^{b} r^{1 / 2}(u) d u, \quad \sigma=\left\{\frac{r(a)}{r(0)}\right\}^{1 / 4}, \\
\rho & =r^{-1 / 4}(0)\left\{r^{-1 / 4}(x)\right\}_{x=a}^{\prime}, \quad \tau=r^{-1 / 4}(0)\left\{r^{-1 / 4}(x)\right\}_{x=0}^{\prime} .
\end{aligned}
$$

Let $\theta(t, \lambda)$ and $\varphi(t, \lambda)$ denote the solutions of problem (1.3), satisfying the initial conditions

$$
\begin{aligned}
\theta(0, \lambda)=1, & \left.\frac{\partial}{\partial t} \theta(t, \lambda)\right|_{t=0}=0 ; \\
\varphi(0, \lambda)=0, & \left.\frac{\partial}{\partial t} \varphi(t, \lambda)\right|_{t=0}=1 .
\end{aligned}
$$

Then, we can define the Hill discriminant (see [1]) of (1.1) by the function

$$
F(\lambda)=\theta(A, \lambda)+\left.\sigma^{2} \frac{\partial}{\partial t} \varphi(t, \lambda)\right|_{t=A}+(\rho \sigma-\tau) \varphi(A, \lambda)
$$

Thus, the eigenvalues of the periodic boundary value problem coincide with the roots $\lambda$ of

$$
F(\lambda)=2 \sigma
$$

and also the eigenvalues of the antiperiodic boundary value problem coincide with the roots of

$$
F(\lambda)=-2 \sigma
$$

Each of the periodic and antiperiodic boundary value problems has a countable infinity real eigenvalues with the points accumulate at $+\infty$. Denote by

$$
\mu_{0}<\mu_{2}^{-} \leq \mu_{2}^{+}<\cdots<\mu_{2 n}^{-} \leq \mu_{2 n}^{+} \cdots
$$

the eigenvalues of the periodic boundary value problem, and by

$$
\mu_{1}^{-} \leq \mu_{1}^{+}<\cdots<\mu_{2 n+1}^{-} \leq \mu_{2 n+1}^{+} \cdots
$$


the eigenvalues of the antiperiodic boundary value problem (the equality holds in the case of double eigenvalue). These values occur in the order

$$
\mu_{0}<\mu_{1}^{-} \leq \mu_{1}^{+}<\mu_{2}^{-} \leq \mu_{2}^{+}<\cdots<\mu_{n}^{-} \leq \mu_{n}^{+}<\cdots .
$$

If $\lambda$ lies in any of the open intervals $\left(-\infty, \mu_{0}\right)$ and $\left(\mu_{n}^{-}, \mu_{n}^{+}\right)(n=1,2, \ldots)$, then all nontrivial solutions of (1.1) are unbounded in $(-\infty, \infty)$. These kind of intervals are called the instability intervals of (1.1). Apart from $\left(-\infty, \mu_{0}\right)$, some or all of the instability intervals vanish for the case of double eigenvalues. If $\lambda$ lies in any of the complementary open intervals $\left(\mu_{n-1}^{+}, \mu_{n}^{-}\right)(n=1,2, \ldots)$, then all solutions of (1.1) are bounded in $(-\infty, \infty)$, and these intervals are called the stability intervals of (1.1). We are interested in the lengths $I_{n}$ of the instability intervals

$$
I_{2 n}=\mu_{2 n}^{+}-\mu_{2 n}^{-}, \quad I_{2 n+1}=\mu_{2 n+1}^{+}-\mu_{2 n+1}^{-} .
$$

Eastham [2] has studied (1.1) where the second derivative of $r(x)$ is piecewise continuous on interval $(0, a)$. But we studied $(1.1)$ where the second derivative of $r(x)$ is piecewise continuous on intervals $(0, b)$ and $(b, a)(0<b<a)$. On the other hands, our method is different from his method and is based on using Rouche's theorem about roots of analytic functions.

\section{An asymptotic formula of the hill discriminant}

Proposition 2.1. Let $\theta(t, \lambda)$ and $\varphi(t, \lambda)$ be the solutions of problem (1.3) such that $\theta(0, \lambda)=1, \dot{\theta}(0, \lambda)=0, \varphi(0, \lambda)=0$, and $\dot{\varphi}(0, \lambda)=1$. For $\lambda=s^{2}$, these solutions verify the following integral equations:

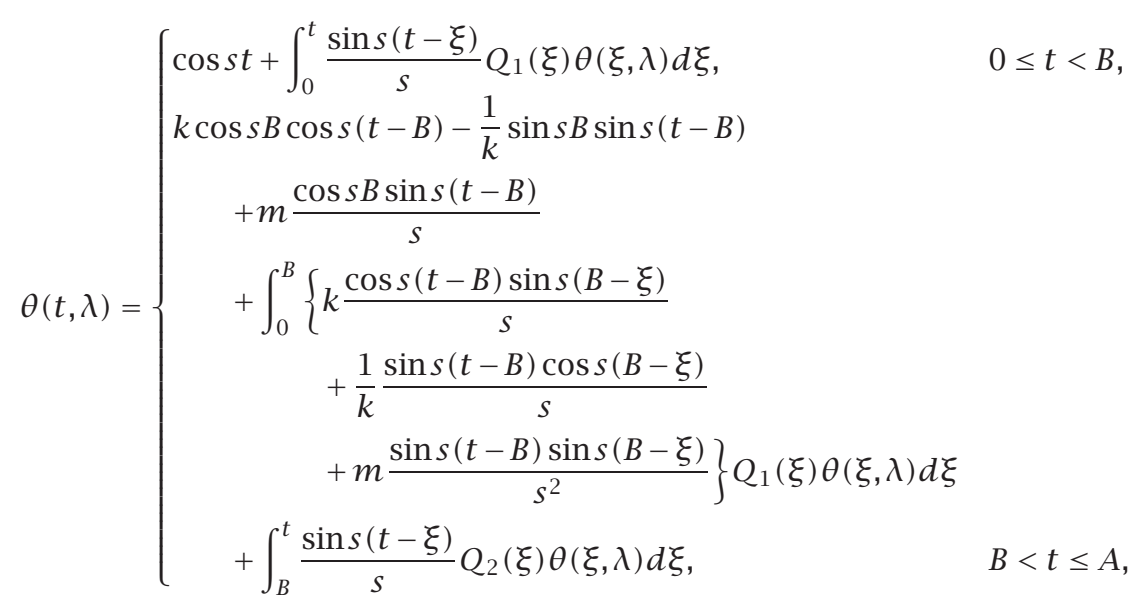




$$
\varphi(t, \lambda)=\left\{\begin{array}{cc}
\frac{\sin s t}{s}+\int_{0}^{t} \frac{\sin s(t-\xi)}{s} Q_{1}(\xi) \varphi(\xi, \lambda) d \xi, & 0 \leq t<B, \\
k \frac{\cos s(t-B) \sin s B}{s}+\frac{1}{k} \frac{\sin s(t-B) \cos s B}{s} & \\
+m \frac{\sin s B \sin s(t-B)}{s^{2}} & \int_{0}^{B}\left\{k \frac{\cos s(t-B) \sin s(B-\xi)}{s}\right. \\
+\frac{1}{k} \frac{\sin s(t-B) \cos s(B-\xi)}{s} & \quad B<t \leq A, \\
\left.+\int_{B}^{t} \frac{\sin s(t-B) \sin s(B-\xi)}{s}\right\} Q_{1}(\xi) \varphi(\xi, \lambda) d \xi & \quad Q_{2}(\xi) \varphi(\xi, \lambda) d \xi,
\end{array}\right.
$$

where $Q_{1}(t)=q(x) / r_{1}(x)-r_{1}^{-3 / 4}(x)\left\{r_{1}^{-1 / 4}(x)\right\}^{\prime \prime}, Q_{2}(t)=q(x) / r_{2}(x)-$ $r_{2}^{-3 / 4}(x)\left\{r_{2}^{-1 / 4}(x)\right\}^{\prime \prime}, k=r^{1 / 4}(b+0) / r^{1 / 4}(b-0), m=\left\{r^{-1 / 4}(b-0)\right\}^{\prime} / r^{1 / 4}(b+$ $0)-\left\{r^{-1 / 4}(b+0)\right\}^{\prime} / r^{1 / 4}(b-0)$.

PROoF. It is clear that $\theta(t, \lambda)$ and $\varphi(t, \lambda)$ verify the integral equations for $0 \leq t<B$.

Let $B<t \leq A$. Since $\theta(t, \lambda)$ and $\varphi(t, \lambda)$ are solutions of problem (1.3), we have

$$
\begin{aligned}
\ddot{\theta}(t, \lambda)+\{\lambda-Q(t)\} \theta(t, \lambda) & =0, \\
\ddot{\varphi}(t, \lambda)+\{\lambda-Q(t)\} \varphi(t, \lambda) & =0 .
\end{aligned}
$$

First, we multiply the equalities above by $\sin s(t-\xi) / s$ and take integral of the obtained equalities from $B$ to $t$. Then, we get

$$
\begin{aligned}
\theta(t, \lambda)= & \cos s(t-B) \theta(B+0, \lambda)+\frac{\sin s(t-B)}{s} \dot{\theta}(B+0, \lambda) \\
& +\int_{B}^{t} \frac{\sin s(t-\xi)}{s} Q_{2}(\xi) \theta(\xi, \lambda) d \xi, \\
\varphi(t, \lambda)= & \cos s(t-B) \varphi(B+0, \lambda)+\frac{\sin s(t-B)}{s} \dot{\varphi}(B+0, \lambda) \\
& +\int_{B}^{t} \frac{\sin s(t-\xi)}{s} Q_{2}(\xi) \varphi(\xi, \lambda) d \xi .
\end{aligned}
$$


Using the equalities $y(b-0)=y(b+0)$ and $y^{\prime}(b-0)=y^{\prime}(b+0)$, we obtain

$$
\begin{gathered}
\theta(B+0, \lambda)=k \theta(B-0, \lambda), \quad \varphi(B+0, \lambda)=k \varphi(B-0, \lambda), \\
\dot{\theta}(B+0, \lambda)=m \theta(B-0, \lambda)+\frac{1}{k} \dot{\theta}(B-0, \lambda), \\
\dot{\varphi}(B+0, \lambda)=m \varphi(B-0, \lambda)+\frac{1}{k} \dot{\varphi}(B-0, \lambda) .
\end{gathered}
$$

When these equalities are written in (2.4), the proof is completed.

Proposition 2.2. Let $\theta(t, \lambda)$ and $\varphi(t, \lambda)$ be the solutions of problem (1.3) such that $\theta(0, \lambda)=1, \dot{\theta}(0, \lambda)=0, \varphi(0, \lambda)=0$, and $\dot{\varphi}(0, \lambda)=1$. Then

$$
\begin{aligned}
F(\lambda)= & k\left\{\cos s(A-B) \cos s B-\sigma^{2} \sin s(A-B) \sin s B\right\} \\
& +\frac{1}{k}\left\{\sigma^{2} \cos s(A-B) \cos s B-\sin s(A-B) \sin s B\right\}+O\left(\frac{e^{|\operatorname{Im} s| A}}{|s|}\right),
\end{aligned}
$$

where $|\lambda| \rightarrow \infty$, that is, $|s| \rightarrow \infty$.

Proof. Using integral equations (2.1) and (2.2), we have

$$
\theta(t, \lambda)= \begin{cases}\cos s t+O\left(\frac{e^{|\operatorname{Im} s| t}}{|s|}\right), & 0 \leq t<B, \\ k \cos s B \cos s(t-B)-\frac{1}{k} \sin s B \sin s(t-B)+O\left(\frac{e^{|\operatorname{Im} s| t}}{|s|}\right), & B<t \leq A,\end{cases}
$$

$\varphi(t, \lambda)= \begin{cases}\frac{\sin s t}{s}+O\left(\frac{e^{|\operatorname{Im} s| t}}{|s|^{2}}\right), & 0 \leq t<B, \\ k \frac{\cos s(t-B) \sin s B}{s}+\frac{1}{k} \frac{\sin s(t-B) \cos s B}{s}+O\left(\frac{e^{|\operatorname{Im} s| t}}{|s|^{2}}\right), & B<t \leq A,\end{cases}$

$$
\dot{\varphi}(t, \lambda)= \begin{cases}\cos s t+O\left(\frac{e^{|\operatorname{Im} s| t}}{|s|}\right), & 0 \leq t<B, \\ -k \sin s B \sin s(t-B)+\frac{1}{k} \cos s B \cos s(t-B)+O\left(\frac{e^{|\operatorname{Im} s| t}}{|s|}\right), & B<t \leq A,\end{cases}
$$

where $|s|$ goes to positive infinity. When we put $\theta(A, \lambda), \varphi(A, \lambda)$, and $\dot{\varphi}(A, \lambda)$ in (1.7), we get the result.

3. The asymptotic formulas for the lengths of the instability intervals. First, we research the eigenvalues of the periodic boundary value problem, using Rouche's theorem which is stated as follows. 
THEOREM 3.1 (Rouche's theorem). If $f(w)$ and $g(w)$ are analytic functions inside and on a simple closed contour $\Gamma$ and $|g(w)|<|f(w)|$ at each point on $\Gamma$, then $f(w)$ and $f(w)+g(w)$ have the same number of zeros, counting multiplicities, inside $\Gamma$.

Let $\sigma=\{r(a) / r(0)\}^{1 / 4}=1$. Then, define

$$
\Phi^{+}(s)=F(\lambda)-2=\frac{k^{2}+1}{k} \cos s A-2+O\left(\frac{e^{|\operatorname{Im} s| A}}{|s|}\right) .
$$

Let $f(s)=\left(\left(k^{2}+1\right) / k\right) \cos s A-2, g(s)=O\left(e^{|\operatorname{Im} s| A} /|s|\right)$ and

$$
\begin{gathered}
\Gamma_{2 n+1 / 2}=\left\{s \in \mathbb{C}:|\operatorname{Re} s|=\frac{1}{A}\left[\left(2 n+\frac{1}{2}\right) \pi+\arccos \frac{2 k}{k^{2}+1}\right],\right. \\
\left.|\operatorname{Im} s|=\frac{1}{A}\left[\left(2 n+\frac{1}{2}\right) \pi+\arccos \frac{2 k}{k^{2}+1}\right]\right\} .
\end{gathered}
$$

In order to apply Rouche's theorem to our case, we need the following lemma.

LEMMA 3.2. There is a positive number $C$ such that

$$
|f(s)| \geq C e^{A|\operatorname{Im} s|}, \quad s \in \Gamma_{2 n+1 / 2},
$$

where $C$ does not depend on $s$ and $n$.

Proof. Let $s=u+i v$. Then

$$
\begin{aligned}
|f(s)|^{2}= & \left|\frac{k^{2}+1}{k} \cos s A-2\right|^{2} \\
= & 4+\frac{1}{4}\left(\frac{k^{2}+1}{k}\right)^{2}\left[e^{2 v A}+e^{-2 v A}+2 \cos 2 u A\right] \\
& -2 \frac{k^{2}+1}{k}\left(e^{v A}+e^{-v A}\right) \cos u A
\end{aligned}
$$

On the vertical edge of square contour $\Gamma_{2 n+1 / 2}$, take

$$
\begin{aligned}
& u=-\left(2 n+\frac{1}{2}\right) \frac{\pi}{A}-\frac{1}{A} \arccos \frac{2 k}{k^{2}+1}, \\
& u=\left(2 n+\frac{1}{2}\right) \frac{\pi}{A}+\frac{1}{A} \arccos \frac{2 k}{k^{2}+1}
\end{aligned}
$$

When the value of $u$ is written in (3.4), we have

$$
|f(s)|^{2} \geq \frac{1}{4}\left(\frac{k^{2}+1}{k}\right)^{2} e^{2|v| A}
$$


and hence

$$
|f(s)| \geq \frac{1}{2}\left(\frac{k^{2}+1}{k}\right) e^{|v| A}
$$

On the other hand, we take that

$$
\begin{aligned}
& v=-\left(2 n+\frac{1}{2}\right) \frac{\pi}{A}-\frac{1}{A} \arccos \frac{2 k}{k^{2}+1}, \\
& v=\left(2 n+\frac{1}{2}\right) \frac{\pi}{A}+\frac{1}{A} \arccos \frac{2 k}{k^{2}+1}
\end{aligned}
$$

on the horizontal edge of square contour $\Gamma_{2 n+1 / 2}$. Since the function $|f(s)|^{2}$ has minimum values at the points $u=p \pi / A$ where $p$ is even, we have

$$
|f(s)|^{2} \geq \frac{1}{16}\left(\frac{k^{2}+1}{k}\right)^{2} e^{2|v| A} .
$$

This completes the proof.

From Lemma 3.2, we have a positive number $C^{\prime}$ such that

$$
\left|\frac{g(s)}{f(s)}\right| \leq \frac{C^{\prime}}{|s|}
$$

For all $s \in \Gamma_{2 n+1 / 2}$,

$$
|s| \geq\left(2 n+\frac{1}{2}\right) \frac{\pi}{A}+\frac{1}{A} \arccos \frac{2 k}{k^{2}+1}
$$

and there exits a natural number $n_{0}$ such that, for all $n \geq n_{0}$,

$$
C^{\prime}<\left(2 n+\frac{1}{2}\right) \frac{\pi}{A}+\frac{1}{A} \arccos \frac{2 k}{k^{2}+1} .
$$

Therefore, for all $s \in \Gamma_{2 n+1 / 2}, n \geq n_{0}$,

$$
\left|\frac{f(s)}{g(s)}\right|<1
$$

Moreover, $f(s)$ and $g(s)$ are analytic on the square contour $\Gamma_{2 n+1 / 2}$ and on the region bounded by the square contour. The number of roots of function $f(s)$ is $4 n+2$ inside the square contour $\Gamma_{2 n+1 / 2}$. By Rouche's theorem, $\Phi^{+}(s)$ has $4 n+2$ roots inside the square contour $\Gamma_{2 n+1 / 2}$. Denote these roots by

$$
-s_{2 n}^{+},-s_{2 n}^{-}, \ldots,-s_{2}^{+},-s_{2}^{-},-s_{0}^{+}, s_{0}^{+}, s_{2}^{-}, s_{2}^{+}, \ldots, s_{2 n}^{-}, s_{2 n}^{+} .
$$


Similarly, $\Phi^{+}(s)$ must have $4 n-2$ roots inside the square contour $\Gamma_{2 n-1 / 2}$. Therefore, there are 4 roots between $\Gamma_{2 n+1 / 2}$ and $\Gamma_{2 n-1 / 2}$. Two of these roots belong to region

$$
\begin{aligned}
D_{n}=\{s \in \mathbb{C} & :\left(2 n-\frac{1}{2}\right) \frac{\pi}{A}+\frac{1}{A} \arccos \frac{2 k}{k^{2}+1}<\operatorname{Re} s \\
& <\left(2 n+\frac{1}{2}\right) \frac{\pi}{A}+\frac{1}{A} \arccos \frac{2 k}{k^{2}+1}, \\
|\operatorname{Im} s| & \left.\leq\left(2 n+\frac{1}{2}\right) \frac{\pi}{A}+\frac{1}{A} \arccos \frac{2 k}{k^{2}+1}\right\} .
\end{aligned}
$$

Consider circles $\left|s-2 n \pi / A \pm(1 / A) \arccos \left(2 k /\left(k^{2}+1\right)\right)\right|=\rho$, where $\rho$ is any number such that $0<\rho<\pi / 2 A$. Then functions $f(s)$ and $g(s)$ are analytic on these circles and region bounded by these circles.

LEMMA 3.3. Consider circles $\left|s-2 n \pi / A \pm(1 / A) \arccos \left(2 k /\left(k^{2}+1\right)\right)\right|=\rho$ $(0<\rho<\pi / 2 A)$. Then, for all $s$ on these circles, there is a positive number $K$ such that

$$
|f(s)| \geq K \rho^{2} e^{A|\operatorname{Im} s|},
$$

where $K$ does not depend on $s$ and $n \in \mathbb{N}$.

Proof. Let $s=u+i v$ and consider $\mid s-2 n \pi / A \pm(1 / A) \arccos \left(2 k /\left(k^{2}+\right.\right.$ 1)) $\mid=\rho$. Then

$$
u=\frac{2 n \pi}{A} \mp \frac{1}{A} \arccos \frac{2 k}{k^{2}+1} \mp \sqrt{\rho^{2}-v^{2}} .
$$

On the other hand,

$$
\begin{aligned}
|f(s)|= & \left(\frac{k^{2}+1}{2 k}\right)\left[e^{v A}+e^{-v A}-\frac{4 k}{k^{2}+1} \cos u A\right] \\
& \times\left[1-\frac{4\left(k^{2}-1\right)^{2}}{\left(k^{2}+1\right)^{2}} \cdot \frac{\sin ^{2} u A}{\left(e^{v A}+e^{-v A}-\left(4 k /\left(k^{2}+1\right)\right) \cos u A\right)^{2}}\right]^{1 / 2} .
\end{aligned}
$$

Let

$$
F(u, v)=\frac{\sin u A}{e^{v A}+e^{-v A}-\left(4 k /\left(k^{2}+1\right)\right) \cos u A} .
$$


This function has a maximum value and a minimum value at the points

$$
\begin{aligned}
& \left(\frac{2 n \pi}{A}+\frac{1}{A} \arccos \frac{2 k}{k^{2}+1}, 0\right), \\
& \left(\frac{2 n \pi}{A}-\frac{1}{A} \arccos \frac{2 k}{k^{2}+1}, 0\right),
\end{aligned}
$$

respectively. So, for all $u$ and $v$ on the circles, we have

$$
2|F(u, v)|<\frac{k^{2}+1}{\left|k^{2}-1\right|}
$$

and hence

$$
0<1-\left[2 \cdot \frac{k^{2}-1}{k^{2}+1} \cdot F(u, v)\right]^{2} .
$$

Therefore, there is a positive number $\epsilon_{0}$ not depending on $\rho$ and $n$ such that

$$
0<\epsilon_{0} \leq 1-\left[2 \cdot \frac{k^{2}-1}{k^{2}+1} \cdot F(u, v)\right]^{2}
$$

Thus, for all $s$ on the circles, we get

$$
|f(s)| \geq L\left[e^{v A}+e^{-v A}-\frac{4 k}{k^{2}+1} \cos u A\right]
$$

where $L=\left(\left(k^{2}+1\right) / 2 k\right) \sqrt{\epsilon_{0}}$. Let $G(u, v)=e^{v A}+e^{-v A}-\left(4 k /\left(k^{2}+1\right)\right) \cos u A$.

CASE 1. Let $u=2 n \pi / A+(1 / A) \arccos \left(2 k /\left(k^{2}+1\right)\right)+\sqrt{\rho^{2}-v^{2}}$ and $u=$ $2 n \pi / A-(1 / A) \arccos \left(2 k /\left(k^{2}+1\right)\right)-\sqrt{\rho^{2}-v^{2}}$. Then,

$$
\begin{aligned}
G(u, v) & =e^{v A}+e^{-v A}-\frac{4 k}{k^{2}+1} \cos u A \\
& \geq e^{v A}+e^{-v A}-\frac{8 k^{2}}{\left(k^{2}+1\right)^{2}} \cos \left(A \sqrt{\rho^{2}-v^{2}}\right) \\
& \geq e^{v A}+e^{-v A}-2 \cos \left(A \sqrt{\rho^{2}-v^{2}}\right)
\end{aligned}
$$

Let $g(v)=e^{v A}+e^{-v A}-2 \cos \left(A \sqrt{\rho^{2}-v^{2}}\right)$. So, we can work on interval $[0, \rho]$ because the function $g$ is even for all $v \in[-\rho, \rho]$. First, take the derivative of $g$ on the variable $v$ and we get

$$
g^{\prime}(v) \geq A\left[e^{v A}-e^{-v A}-2 A v\right]>0
$$


So, $g$ is increasing and then $g(0) \leq g(v)$ for $0 \leq v \leq \rho$. Moreover,

$$
0 \leq A \rho \leq \frac{\pi}{2} \Longrightarrow \frac{\sqrt{2} A \rho}{\pi} \leq \sin \frac{A \rho}{2}
$$

and hence

$$
8\left(\frac{A \rho}{\pi}\right)^{2} \leq 4 \sin ^{2} \frac{A \rho}{2} \leq g(v)
$$

Since $|f(s)| \geq 8 A^{2} \rho^{2} L / \pi^{2}$, we have

$$
\frac{e^{A|v|}}{|f(s)|} \leq \frac{e^{A|v|}}{8 A^{2} \rho^{2} L / \pi^{2}} \leq \frac{1}{K \rho^{2}}
$$

where $K=8 A^{2} \rho^{2} L / \pi^{2} e^{\pi / 2}$. Hence, $|f(s)| \geq K \rho^{2} e^{A|v|}$.

CASE 2. Similarly, when we take

$$
\begin{aligned}
& u=\frac{2 n \pi}{A}+\frac{1}{A} \arccos \frac{2 k}{k^{2}+1}-\sqrt{\rho^{2}-v^{2}} \\
& u=\frac{2 n \pi}{A}-\frac{1}{A} \arccos \frac{2 k}{k^{2}+1}+\sqrt{\rho^{2}-v^{2}}
\end{aligned}
$$

the inequality is verified.

From Lemma 3.3 and definition of $g(s)$, we have

$$
\left|\frac{g(s)}{f(s)}\right| \leq \frac{C_{1}\left(e^{A|y|} /|s|\right)}{K \rho^{2} e^{A|y|}}
$$

We can choose $\rho=\sqrt{2 C_{2} /\left((2 n-1 / 2) \pi / A+(1 / A) \arccos \left(2 k /\left(k^{2}+1\right)\right)\right)}$, where $C_{2}=C_{1} / K$ because there is a positive number $m$ such that for all $n \geq m$, $\rho<\pi / 2 A$. Moreover, for all $s$ on these circles, we have

$$
|s| \geq\left(2 n-\frac{1}{2}\right) \frac{\pi}{A}+\frac{1}{A} \arccos \frac{2 k}{k^{2}+1} .
$$

Thus,

$$
\left|\frac{g(s)}{f(s)}\right| \leq \frac{C_{2}}{\rho^{2}\left[(2 n-1 / 2) \pi / A+(1 / A) \arccos \left(2 k /\left(k^{2}+1\right)\right)\right]}=\frac{1}{2}<1 .
$$


By Rouche's theorem, the function $\Phi^{+}(s)$ has one root inside the circle

$$
\left|s-\frac{2 n \pi}{A}+\frac{1}{A} \arccos \frac{2 k}{k^{2}+1}\right|=\rho
$$

and one root inside the circle

$$
\left|s-\frac{2 n \pi}{A}-\frac{1}{A} \arccos \frac{2 k}{k^{2}+1}\right|=\rho .
$$

Denote these roots by $s_{2 n}^{-}$and $s_{2 n}^{+}$, respectively. Let

$$
\begin{aligned}
& s_{2 n}^{-}=\frac{2 n \pi}{A}-\frac{1}{A} \arccos \frac{2 k}{k^{2}+1}+\frac{r_{2 n}^{-}}{\sqrt{n}}, \\
& s_{2 n}^{+}=\frac{2 n \pi}{A}+\frac{1}{A} \arccos \frac{2 k}{k^{2}+1}+\frac{r_{2 n}^{+}}{\sqrt{n}} .
\end{aligned}
$$

Since $\left|r_{2 n}^{\mp}\right|<\sqrt{2 n C_{2} /\left((2 n-1 / 2) \pi / A+(1 / A) \arccos \left(2 k /\left(k^{2}+1\right)\right)\right)}$, we have $\operatorname{Sup} r_{2 n}^{\mp}<\infty$. Hence, for periodic boundary value problem, we have

$$
\begin{aligned}
& s_{2 n}^{-}=\frac{2 n \pi}{A}-\frac{1}{A} \arccos \frac{2 k}{k^{2}+1}+O\left(\frac{1}{\sqrt{n}}\right), \\
& s_{2 n}^{+}=\frac{2 n \pi}{A}+\frac{1}{A} \arccos \frac{2 k}{k^{2}+1}+O\left(\frac{1}{\sqrt{n}}\right) .
\end{aligned}
$$

Similarly, for antiperiodic boundary value problem, we have

$$
\begin{aligned}
& s_{2 n+1}^{-}=\frac{(2 n+1) \pi}{A}-\frac{1}{A} \arccos \frac{2 k}{k^{2}+1}+O\left(\frac{1}{\sqrt{n}}\right), \\
& s_{2 n+1}^{+}=\frac{(2 n+1) \pi}{A}+\frac{1}{A} \arccos \frac{2 k}{k^{2}+1}+O\left(\frac{1}{\sqrt{n}}\right) .
\end{aligned}
$$

Combining these two results, we get

$$
\begin{aligned}
& s_{n}^{-}=\frac{n \pi}{A}-\frac{1}{A} \arccos \frac{2 k}{k^{2}+1}+O\left(\frac{1}{\sqrt{n}}\right), \\
& s_{n}^{+}=\frac{n \pi}{A}+\frac{1}{A} \arccos \frac{2 k}{k^{2}+1}+O\left(\frac{1}{\sqrt{n}}\right) .
\end{aligned}
$$


THEOREM 3.4. Let $r^{\prime}(0) \neq r^{\prime}(a)$. If the second derivative of function $r(x)$ in (1.1) is piecewise continuous on open intervals $(0, b)$ and $(b, a)$, then

$$
I_{n}=\frac{4 n \pi}{A^{2}} \arccos \frac{2 k}{k^{2}+1}+O(1)
$$

PROoF. We know that eigenvalues of periodic and antiperiodic boundary value problems are real and go to infinity. So, it is sufficient to take positive values of parameters $\lambda$. By (2.7) and (2.8), we have

$$
\begin{gathered}
\theta(t, \lambda)= \begin{cases}\cos s t+O\left(\frac{1}{|s|}\right), & \text { if } 0 \leq t<B, \\
k \cos s(t-B) \cos s B-\frac{1}{k} \sin s(t-B) \sin s B+O\left(\frac{1}{|s|}\right), & \text { if } B<t \leq A,\end{cases} \\
\varphi(t, \lambda)= \begin{cases}\frac{\sin s t}{s}+O\left(\frac{1}{|s|^{2}}\right), & \text { if } 0 \leq t<B, \\
k \frac{\cos s(t-B) \sin s B}{s}+\frac{1}{k} \frac{\sin s(t-B) \cos s B}{s}+O\left(\frac{1}{|s|^{2}}\right), & \text { if } B<t \leq A .\end{cases}
\end{gathered}
$$

When the values $\theta(t, \lambda)$ and $\varphi(t, \lambda)$ are written in (2.1) and in derivative of (2.2), we get

$$
\begin{aligned}
\theta(A, \lambda)= & k \cos s(A-B) \cos s B-\frac{1}{k} \sin s(A-B) \sin s B+m \frac{\cos s B \sin s(A-B)}{s} \\
& +\frac{1}{4 s} k \int_{0}^{B}[\sin s A-\sin s(A-2 B)+\sin s(A-2 \xi)-\sin s(A-2 B+2 \xi)] \\
& \quad \times Q_{1}(\xi) d \xi \\
+ & \frac{1}{4 s} \frac{1}{k} \int_{0}^{B}[\sin s A+\sin s(A-2 B)+\sin s(A-2 \xi)+\sin s(A-2 B+2 \xi)] \\
& +\frac{1}{4 s} k \int_{B}^{A}[\sin s A+\sin s(A-2 B)+\sin s(A-2 \xi)+\sin s(A+2 B-2 \xi)] \\
& \times Q_{2}(\xi) d \xi \\
+ & \frac{1}{4 s} \frac{1}{k} \int_{B}^{A}[\sin s A-\sin s(A-2 B)+\sin s(A-2 \xi)-\sin s(A+2 B-2 \xi)] \\
& \times Q_{2}(\xi) d \xi \\
+ & O\left(\frac{1}{|s|^{2}}\right),
\end{aligned}
$$




$$
\begin{aligned}
\dot{\varphi}(A, \lambda)= & -k \sin s(A-B) \sin s B+\frac{1}{k} \cos s(A-B) \cos s B+m \frac{\sin s B \cos s(A-B)}{s} \\
& +\frac{1}{4 s} k \int_{0}^{B}[\sin s A+\sin s(A-2 B)-\sin s(A-2 \xi)-\sin s(A-2 B+2 \xi)] \\
& \times Q_{1}(\xi) d \xi \\
& +\frac{1}{4 s} \frac{1}{k} \int_{0}^{B}[\sin s A-\sin s(A-2 B)-\sin s(A-2 \xi)+\sin s(A-2 B+2 \xi)] \\
& +\frac{1}{4 s} k \int_{B}^{A}[\sin s A-\sin s(A-2 B)-\sin s(A-2 \xi)+\sin s(A+2 B-2 \xi)] \\
& +\frac{1}{4 s} \frac{1}{k} \int_{B}^{A}[\sin s A+\sin s(A-2 B)-\sin s(A-2 \xi)-\sin s(A+2 B-2 \xi)] \\
& +O\left(\frac{1}{|s|^{2}}\right) .
\end{aligned}
$$

Moreover, we have

$$
\varphi(A, \lambda)=k \frac{\cos s(A-B) \sin s B}{s}+\frac{1}{k} \frac{\sin s(A-B) \cos s B}{s}+O\left(\frac{1}{|s|^{2}}\right) .
$$

After putting the values $\theta(A, \lambda), \varphi(A, \lambda)$, and $\dot{\varphi}(A, \lambda)$ in equality

$$
\theta(A, \lambda)+\dot{\varphi}(A, \lambda)+(\rho-\tau) \varphi(A, \lambda)=2,
$$

we get

$$
\begin{aligned}
\cos s_{2 n}^{\mp} A & +\frac{1}{s_{2 n}^{\mp}} \cdot \frac{k m}{k^{2}+1} \sin s_{2 n}^{\mp} A \\
& +\frac{1}{2 s_{2 n}^{\mp}} \sin s_{2 n}^{\mp} A \int_{0}^{B} Q_{1}(\xi) d \xi+\frac{1}{2 s_{2 n}^{\mp}} \sin s_{2 n}^{\mp} A \int_{B}^{A} Q_{2}(\xi) d \xi \\
& -\frac{1}{2 s_{2 n}^{\mp}} \cdot \frac{k^{2}-1}{k^{2}+1} \int_{0}^{B} \sin s_{2 n}^{\mp}(A-2 B+2 \xi) Q_{1}(\xi) d \xi \\
& +\frac{1}{2 s_{2 n}^{\mp}} \cdot \frac{k^{2}-1}{k^{2}+1} \int_{B}^{A} \sin s_{2 n}^{\mp}(A+2 B-2 \xi) Q_{2}(\xi) d \xi \\
& +\frac{\rho-\tau}{2 s_{2 n}^{\mp}} \sin s_{2 n}^{\mp} A-\frac{\rho-\tau}{2 s_{2 n}^{\mp}} \cdot \frac{k^{2}-1}{k^{2}+1} \sin s_{2 n}^{\mp}(A-2 B) \\
& -\frac{2 k}{k^{2}+1}+O\left(\frac{1}{\left|s_{2 n}^{\mp}\right|^{2}}\right)=0 .
\end{aligned}
$$

Let $w=\arccos \left(2 k /\left(k^{2}+1\right)\right)$ and $\delta_{2 n}^{\mp}=O(1 / \sqrt{n})$. Then

$$
s_{2 n}^{\mp} A=2 n \pi \mp \omega+\delta_{2 n}^{\mp} .
$$


Since $1 / s_{2 n}^{\mp}=O(1 / n), 1 /\left(s_{2 n}^{\mp}\right)^{2}=O\left(1 / n^{2}\right), \int_{0}^{B} Q_{1}(\xi) d \xi<\infty$, and $\int_{B}^{A} Q_{2}(\xi) d \xi<$ $\infty$, (3.45) becomes

$$
\mp \delta_{2 n}^{\mp} \sin w=O\left(\frac{1}{n}\right)
$$

it is clear that $\sin w \neq 0$. Hence, we have $\delta_{2 n}^{\mp}=O(1 / n)$ and therefore

$$
\begin{gathered}
I_{2 n}=\mu_{2 n}^{+}-\mu_{2 n}^{-}=\frac{8 n \pi}{A^{2}} \arccos \frac{2 k}{k^{2}+1}+O(1), \\
I_{2 n+1}=\mu_{2 n+1}^{+}-\mu_{2 n+1}^{-}=\frac{4(2 n+1) \pi}{A^{2}} \arccos \frac{2 k}{k^{2}+1}+O(1) .
\end{gathered}
$$

This completes the proof.

THEOREM 3.5. Under hypotheses of Theorem 3.4, we have

$$
\begin{aligned}
I_{n}= & 4 n \pi \omega A^{-2}+A^{-1} \frac{k^{2}-1}{k^{2}+1} \\
& \cdot \frac{2}{\sin \omega}\left\{\int_{0}^{B} \cos \left[\frac{2 \omega}{A}(B-\xi)-\omega\right] \cdot \sin \left[\frac{2 n \pi}{A}(B-\xi)\right] Q_{1}(\xi) d \xi\right. \\
& +\int_{B}^{A} \cos \left[\frac{2 \omega}{A}(B-\xi)+\omega\right] \cdot \sin \left[\frac{2 n \pi}{A}(B-\xi)\right] Q_{2}(\xi) d \xi \\
& \left.+(\rho-\tau) \cos \left[\frac{2 B \omega}{A}-\omega\right] \cdot \sin \left[2 n \pi \frac{B}{A}\right]\right\} \\
& +O\left(\frac{1}{n}\right) .
\end{aligned}
$$

Proof. Since $s_{2 n}^{\mp} A=2 n \pi \mp \omega+\delta_{2 n}^{\mp}$ and $\delta_{2 n}^{\mp}=O(1 / n)$, we have

$$
\begin{gathered}
\cos s_{2 n}^{\mp} A=\cos \omega \pm \delta_{2 n}^{\mp} \sin \omega+O\left(\left(\delta_{2 n}^{\mp}\right)^{2}\right), \quad \sin s_{2 n}^{\mp} A=\mp \sin \omega+O\left(\delta_{2 n}^{\mp}\right), \\
\sin s_{2 n}^{\mp}(A-2 B+2 \xi)=\sin \left[\frac{2}{A}(-B+\xi)(2 n \pi \mp \omega) \mp \omega\right]+O\left(\delta_{2 n}^{\mp}\right), \\
\sin s_{2 n}^{\mp}(A+2 B-2 \xi)=\sin \left[\frac{2}{A}(B-\xi)(2 n \pi \mp \omega) \mp \omega\right]+O\left(\delta_{2 n}^{\mp}\right), \\
\sin s_{2 n}^{\mp}(A-2 B)=-\sin \left[\frac{2 B}{A}(2 n \pi \mp \omega) \pm \omega\right]+O\left(\delta_{2 n}^{\mp}\right), \\
\frac{1}{s_{2 n}^{\mp}}=\frac{A}{2 n \pi}+O\left(\frac{1}{n^{2}}\right) .
\end{gathered}
$$


Using these equalities in (3.45), we get

$$
\begin{aligned}
\delta_{2 n}^{\mp}= & \frac{A}{2 n \pi} \cdot \frac{k m}{k^{2}+1}+\frac{A}{4 n \pi} \int_{0}^{B} Q_{1}(\xi) d \xi \\
& \mp \frac{A}{4 n \pi} \cdot \frac{k^{2}-1}{k^{2}+1} \cdot \frac{1}{\sin \omega} \int_{0}^{B} \sin \left[\frac{2}{A}(B-\xi)(2 n \pi \mp \omega) \pm \omega\right] Q_{1}(\xi) d \xi \\
& \mp \frac{A}{4 n \pi} \cdot \frac{k^{2}-1}{k^{2}+1} \cdot \frac{1}{\sin \omega} \int_{B}^{A} \sin \left[\frac{2}{A}(B-\xi)(2 n \pi \mp \omega) \mp \omega\right] Q_{2}(\xi) d \xi \\
& +\frac{A}{4 n \pi} \int_{B}^{A} Q_{2}(\xi) d \xi \mp \frac{A}{4 n \pi}(\rho-\tau) \cdot \frac{k^{2}-1}{k^{2}+1} \cdot \frac{\sin [(2 B / A)(2 n \pi \mp \omega) \pm \omega]}{\sin \omega} \\
& +\frac{A}{4 n \pi}(\rho-\tau)+O\left(\frac{1}{n^{2}}\right) .
\end{aligned}
$$

Therefore,

$$
\begin{aligned}
I_{2 n}= & 8 n \pi \omega A^{-2}+A^{-1} \frac{k^{2}-1}{k^{2}+1} \\
& \cdot \frac{2}{\sin \omega}\left\{\int_{0}^{B} \cos \left[\frac{2 \omega}{A}(B-\xi)-\omega\right] \cdot \sin \left[\frac{4 n \pi}{A}(B-\xi)\right] Q_{1}(\xi) d \xi\right. \\
& +\int_{B}^{A} \cos \left[\frac{2 \omega}{A}(B-\xi)+\omega\right] \cdot \sin \left[\frac{4 n \pi}{A}(B-\xi)\right] Q_{2}(\xi) d \xi \\
& \left.+(\rho-\tau) \cos \left[\frac{2 B \omega}{A}-\omega\right] \cdot \sin \left[4 n \pi \frac{B}{A}\right]\right\} \\
& +O\left(\frac{1}{n}\right) .
\end{aligned}
$$

Similarly,

$$
\begin{aligned}
I_{2 n+1}= & 4(2 n+1) \pi \omega A^{-2}+A^{-1} \frac{k^{2}-1}{k^{2}+1} \\
& \cdot \frac{2}{\sin \omega}\left\{\int_{0}^{B} \cos \left[\frac{2 \omega}{A}(B-\xi)-\omega\right] \cdot \sin \left[\frac{(4 n+2) \pi}{A}(B-\xi)\right] Q_{1}(\xi) d \xi\right. \\
& +\int_{B}^{A} \cos \left[\frac{2 \omega}{A}(B-\xi)+\omega\right] \cdot \sin \left[\frac{(4 n+2) \pi}{A}(B-\xi)\right] Q_{2}(\xi) d \xi \\
& \left.+(\rho-\tau) \cos \left[\frac{2 B \omega}{A}-\omega\right] \cdot \sin \left[(4 n+2) \pi \frac{B}{A}\right]\right\} \\
& +O\left(\frac{1}{n}\right) .
\end{aligned}
$$

This completes the proof. 


\section{REFERENCES}

[1] M. S. P. Eastham, The Spectral Theory of Periodic Differential Equations, Scottish Academic Press, Edinburgh, 1973.

[2] _ Results and problems in the spectral theory of periodic differential equations, Spectral Theory and Differential Equations (Proc. Sympos., Dundee, 1974, Dedicated to Konrad Jörgens), Lecture Notes in Mathematics, vol. 448, Springer, Berlin, 1975, pp. 126-135.

Ilkay Yaslan Karaca: Department of Mathematics, Faculty of Science, Ege University, Izmir 35100, Turkey

E-mail address: yas1an@sci .ege.edu.tr 


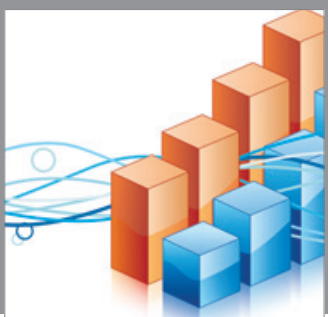

Advances in

Operations Research

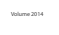

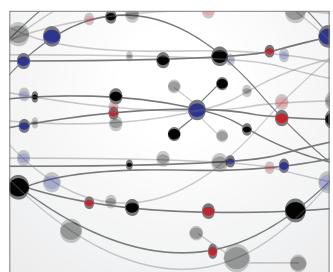

\section{The Scientific} World Journal
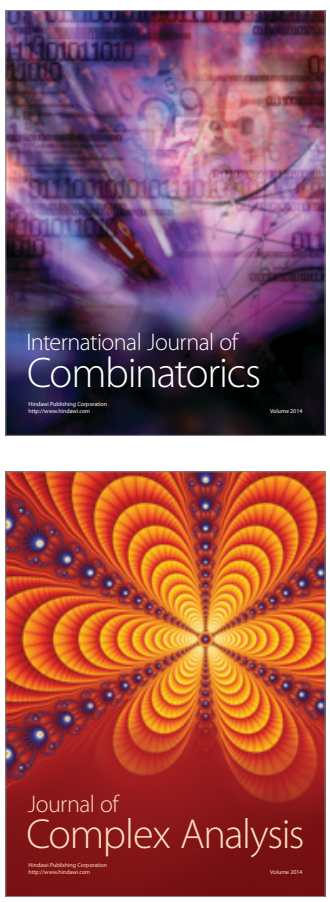

International Journal of

Mathematics and

Mathematical

Sciences
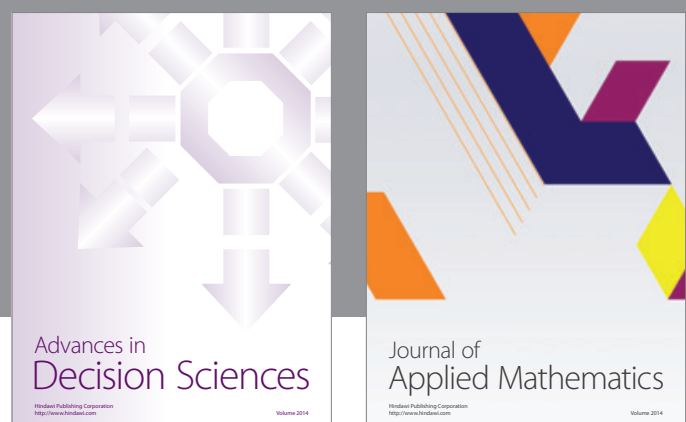

Journal of

Applied Mathematics
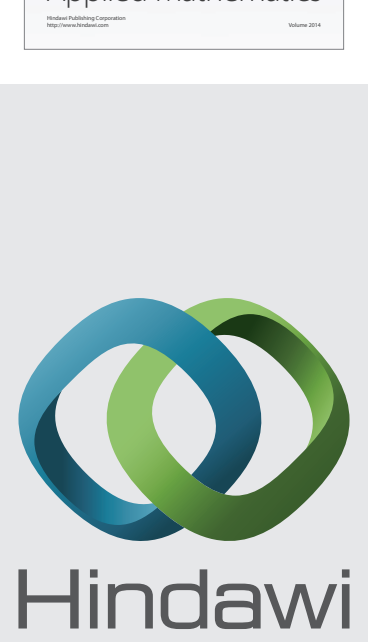

Submit your manuscripts at http://www.hindawi.com
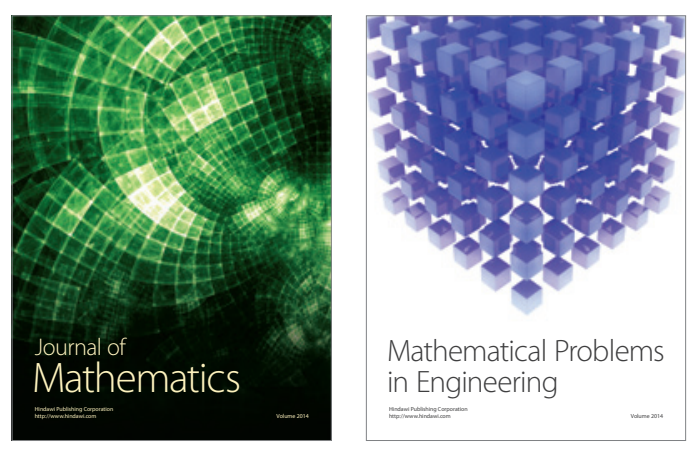

Mathematical Problems in Engineering
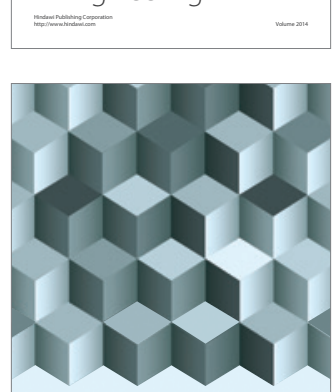

Journal of

Function Spaces
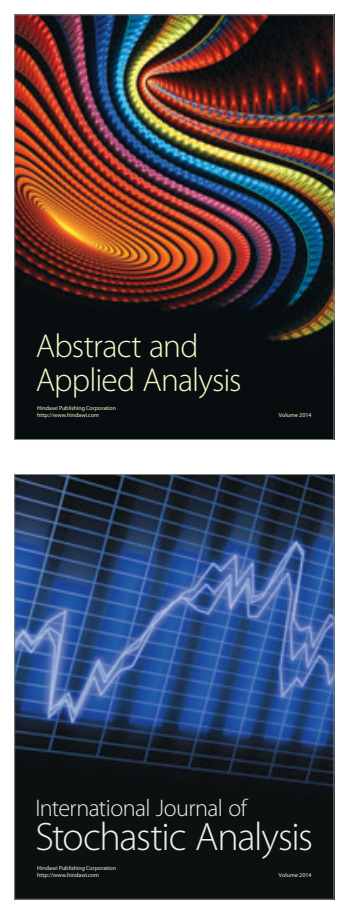

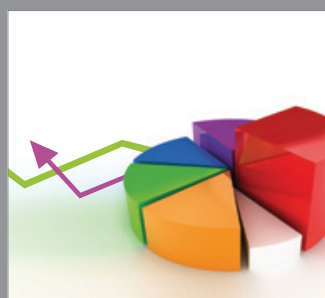

ournal of

Probability and Statistics

Promensencen
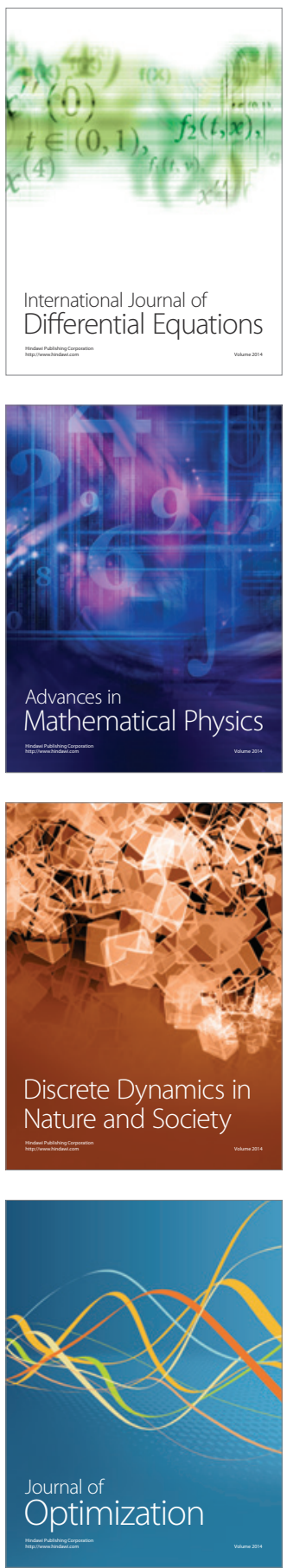